\title{
Efectos de la pentoxifilina sobre la movilidad espermática en pacientes normo y oligoastenospérmicos y su relación con la fecundación in vitro
}

\author{
Díaz I.; Wolf Jean P.; Bulwa S.; Rodrigues D.; Corsetti M.; Jouannet P.
}

RESUMEN OBJETIVO: Determinar los efectos de la incubación de espermatozoides (spz) con Pentoxifilina (PF) sobre las características de movimiento de pacientes normo y oligoastenospérmicos y el resultado de su utilización en fecundación in vitro (FIV) por oligoastenospermia severa.

MATERIAL Y METODOS: Sobre un total de 46 pacientes divididos en tres grupos: Grupo 1 (n=10) normospérmicos; Grupo 2a (n=14) oligoastenospermia moderada y Grupo $2 b(n=22)$ oligoastenospermia severa se cuantificaron los parámetros de movimiento usando computer-assited sperm analysis (CASA). Una segunda parte del estudio consistió en la evaluación del resultado de fecundación in vitro (FIV) en 16 parejas del grupo 2b. Los resultados de FIV e inseminación subzonal (SUZI) con o sin PF fueron comparados.

PREPARACION DEL SEMEN: EI semen fue preparado con gradiente de percoll y dividido en dos partes, uno como control y la otra mitad fue incubada con pentoxifilina $1 \mathrm{mg} / \mathrm{ml}$ después de dos horas de capacitación. Luego de remover la PF del semen, fue analizado con CASA y tres horas después.

RESULTADOS: Se encontró un incremento significativo del desplazamiento lateral de la cabeza (ALH) solamente en el grupo 2a después de la utilización de la PF $(\mathbf{P}<\mathbf{0 . 0 5})$. En los otros dos grupos ningún efecto superior significativo de los parámetros de movimiento fue observado en comparación a una preparación solamente de percoll. Ningún aumento de la taza de fecundación fue observada cuando el semen tratado con PF fue usado comparado con el semen control.

CONCLUSIONES: Aunque la pentoxifilina puede mejorar los parámetros de movimiento en algunos pacientes con oligoastenospermia, dicho tratamiento no supera la capacidad fecundante cuando el semen ha sido previamente seleccionado con gradiente de percoll. Su utilidad en FIV por infertilidad masculina deberá ser evaluada en un grupo más amplio.

PALABRAS ClaVES: Pentoxifilina, fecundación in vitro, inseminación subzonal, movimiento espermático, oligoastenospermia.

SUMMARY OBJECTIVE: To evaluate pentoxifylline effects on sperm movement parameters of normo and oligospermic patients and whether it can improve their fertilizing ability.

MATERIAL AND METHODS: Forty six patients were included in the study, 10 normospermic, 14 moderately oligospermic and 22 severely oligospermic.

DESING: Spermatozoa were selected on percoll gradient, incubated in B2 medium and aliquoted in two parts, one control and one being treated by pentoxifylline $1 \mathrm{mg} / \mathrm{ml}$ after 2 hours of capacitation. Sperm movement parameters were evaluated using computer-assisted sperm analysis (CASA) after selection and after 3 hours of incubation. IVF and SUZI results were compared on sibling oocyte with and without pentoxifylline treatment of the spermatozoa for 16 men who were severely oligospermic.

RESULTS: Pentoxifylline coincubation dit not increase the percentage of motile spermatozoa. It had no effect on the sperm movement parameters of normospermic patients. It significantly increased ALH $(\mathrm{P}<0.05)$ of oligospermic patients. No improvement of the fertilization rate was noticed when pentoxifylline treated sperm were used compared to no treated selected spermatozoa.

CONCLUSIONS: Although pentoxifylline can improve sperm movement in some patients with oligospermia, such a treatment is not useful for improving fertilizing ability when sperm are previously selected on minipercoll gradient.

KEY WORDS: Pentoxifylline, in vitro fertilization, subzonal insemination, sperm movement, oligospermic.

* Laboratoire de Biologie de la Reproduction et du Développement - Histologie, embryologie, Cytogénétique. Hôpital Bicetre, 78, rue du Général Leclerc, Kremlin Bicetre 94275 France. Tel: (33) 145212320 . Fax (33) 145212856.

\section{Introducción}

La pentoxifilina (1-(5-oxohexyl)-3.7-dimetilxantina) es un inhibidor de la fosfodiesterasa, produciendo incremento intracelular de AMPc y GMPc del espermatozoide (1) mecanismo propuesto para mejorar la movilidad 
espermática. Diferentes efectos de la pentoxifilina sobre los parámetros de movimiento espermático y/o motilidad del espermatozoide han sido reportados. Yovih et al. (2) demostraron un aumento del porcentaje de la motilidad de espermatozoides de pacientes astenospérmicos al utilizar pentoxifilina después de selección por migración (Swim-up). Tesarik et al. (3) encontraron un incremento significativo de todos los parámetros del movimiento espermático evaluado por computer-assited sperm analysis (CASA) para pacientes normo y astenospérmicos después de la incubación de espermatozoides capacitados con pentoxifilina $(3.6 \mathrm{mM})$, pero la pentoxifilina no modificó el porcentaje de motilidad espermática. Además, Lewis et al. (4) encontraron únicamente un incremento significativo de la velocidad curvilínea (VCL), del desplazamiento lateral de la cabeza (ALH) y de la frecuencia de Batido (BCF) sobre los espermatozoides seleccionados por swim-up de pacientes normospérmicos después de la incubación con pentoxifilina $(3.6 \mathrm{mM})$. Pang et al (5) únicamente reporta un incremento significativo del porcentaje de hiperactivación espermática cuando espermatozoides astenospérmicos fueron incubados con pentoxifilina por una hora después de ser lavados con Hepes-buffered Ham's F-10.

La capacidad fecundante del espermatozoide ha sido correlacionada con sus características de movimiento (67). Sí la pentoxifilina mejora el movimiento espermático podría entonces incrementar la taza de fertilización cuando fuera usada en pacientes con infertilidad masculina. Sin embargo, resultados discordantes han sido reportados. Usando pentoxifilina en 9 pacientes con infertilidad masculina y fracasos previos de fecundación in vitro. Yovich et al. (8) reporta un incremento moderado de la taza de fertilización de $17.2 \%$ a $22.7 \%$.

Por el contrario, Tournaye et al. (9) no encontró un incremento de la taza de fertilización y de embarazo en un grupo de 22 parejas con infertilidad masculina y fracasos previos de fecundación in vitro.

El propósito de este estudio es evaluar el efecto de la pentoxifilina sobre los parámetros de movimiento espermático y capacidad fecundante del espermatozoide usando fecundación in vitro standard o inseminación subzonal (SUZI).

\section{Materiales y métodos}

\section{Pacientes}

Cuarenta y seis pacientes infértiles que consultaron al laboratorio para análisis rutinario de semen fueron incluidos en este estudio. El semen fue analizado de acuerdo con los criterios de la Organización Mundial de la Salud (10). Los pacientes del grupo $1(n=10)$ tenían $>10.10^{6}$ espermatozoides móviles $/ \mathrm{ml}$ y fueron llamados normospérmicos. Pacientes del grupo 2 tenían $<10.10^{6}$ espermatozoides móviles/ml y fueron llamados oligoastenospérmicos. Nosotros dividimos este último grupo 2a como moderadamente oligoastenospérmicos ( $>5$ y $<10.10^{6}$ espermatozoides móviles/ml, $\mathrm{n}=14$ ); y grupo $2 \mathrm{~b}$ como severamente oligoastenospérmicos $\left(<5.10^{6}\right.$ espermatozoides móviles/ml, $\mathrm{n}=22$ ). En todos los casos, el test de penetración in vitro y en moco cervical fue positivo (11) y no presentaban anticuerpos antiespermatozoides (12); 16 parejas infértiles del grupo $2 \mathrm{~b}$ fueron incluidos en el programa de fecundación in vitro. Ellos presentaban una infertilidad entre 3 y 15 años. Las edades de hombres y mujeres se encontraban entre 28 a 40 y 26 a 37 años respectivamente.

\section{Preparación espermática}

El semen fue recolectado por masturbación después de una abstinencia sexual de 3 a 5 días. El eyaculado se dejó para licuefacción por $30 \mathrm{~min}$ a $37^{\circ} \mathrm{C}$. Después fue colocado a centrifugación en gradiente de percoll $(47.5 \%$ y $95 \%$ ) (Pharmacia, France) por $20 \mathrm{~min}$ a $300 \mathrm{~g}$. El sedimento es removido y lavado nuevamente por centrifugación ( $5 \mathrm{~min}, 600 \mathrm{~g}$ ) en $5 \mathrm{ml}$ de B2 medium (Bio Mérieux, France). Los espermatozoides seleccionados fueron divididos en dos partes. Uno fue incubado a temperatura ambiente y sirvió como control. Después de 2 horas, la pentoxifilina (concentración final $1 \mathrm{mg} / \mathrm{ml}=$ $3.6 \mathrm{mM}$ ) fue adicionada a la segunda mitad por $40 \mathrm{~min}$. La pentoxifilina fue removida por lavado con B2 medium $(5 \mathrm{ml})$ y centrifugación $(5 \mathrm{~min} ; 600 \mathrm{~g})$. El sedimento fue resuspendido en $0.2 \mathrm{ml}$ de $\mathrm{B} 2$.

$\mathrm{El}$ análisis de movimiento espermático fue realizado en todos los aliquotas después de incubación con pentoxifilina (TO) y después de tres horas (T3) de incubación a $37^{\circ} \mathrm{C}$ en aire/CO $(5 \%)$ usando computer assisted analyser system (HTM 2030 versión 7.2; HamiltonThorn Research, Danvers, MA).

Una dilución fue realizada para obtener una concentración final de $10^{6}$ espermatozoides $/ \mathrm{ml}$ e introducida en un tubo microcapilar plano de $200 \mu \mathrm{m}$ de profundidad (Vitro Dynamics, Rockaway, us). Un mínimo de 150 espermatozoides por análisis fueron evaluados. Los parámetros de movimiento espermático fueron velocidad de trayecto (VAP), velocidad curvilinea (VCL), velocidad progresiva (VSL), linearilidad $(\mathrm{LIN}=\mathrm{VSL} / \mathrm{VCL})$, rectitud (STR $=$ VSL/VAP), desplazamiento lateral de la cabeza (ALH), frecuencia de batido $(B C F)$ y porcentaje de hiperactivación (HYPERACT).

\section{Ovocitos e inseminación por FIV y SUZI}

El crecimiento folicular fue estimulado con gonadotrofina menopáusica humana (hMG) en conjunto con análogos de GnRH. La ovulación fue inducida con 10.000 IU de gonadotrofina coriónica humana (hCG) y los ovocitos fueron obtenidos 36 horas después por ecografía transvaginal (13). El cumulus oophorus fue lavado e individualmente desplazado dentro de $30 \mu \mathrm{l}$ de B2 medium bajo aceite equilibrado y conservado en incubadora a $37^{\circ} \mathrm{C}$ con aire $/ \mathrm{CO}_{2} 5 \%$. Cuando la inseminación subzonal (SUZI) fue indicada, las células del cumulus fueron removidas con $0.1 \%$ hialuronidasa (Type III, Sigma, St Louis, MO) en B2 medium.

La decisión de inseminación tipo fecundación in vitro standard o tipo SUZI fue en base al siguiente criterio. 
Cuando la suspensión obtenida después de selección percoll contenía más de 500 espermatozoides móviles por $\mu \mathrm{l}, 5.000$ espermatozoides fueron inseminados en una gota de $30 \mu \mathrm{l}$ de $\mathrm{B} 2$ conteniendo un complejo de cumulus oophorus. Cuando la concentración de espermatozoides seleccionados fue menos de 500 espermatozoides móviles por $\mu$ l o cuando la pareja había tenido fracaso previo de FIV, los ovocitos fueron microinyectados.

En todos los casos la mitad de los ovocitos fueron tratados con semen control y mitad con semen incubado con pentoxifilina. Se recuperaron 186 ovocitos de un total de 16 ciclos; 143 fueron inseminados por procedimiento standard y 37 por SUZI. La incubación con pentoxifilina fue hecha justo antes de la inseminación o SUZI como previamente se ha descrito.

La inseminación subzonal descrita por Wolf J. Ph. et al. (14) fue ejecutada usando dos micromanipuladores Narishige y un microscopio invertido Olympus IMT-2. El número de espermatozoides microinyectados varió entre 3 a 10.

\section{Embriones}

Los ovocitos fueron visualizados 16-18 horas después de inseminación o SUZI para evidencia de fertilización. Los ovocitos que presentaban dos pronúcleos fueron considerados como fertilizados normalmente. Unicamente los embriones con división regular fueron transferidos utilizando catéter de Frydman (Prodimed, Nevilly-en-Thelle, france).

\section{Análisis estadístico}

Todos los datos fueron analizados con Macintosh (R) (Apple Computer). Diferencias de parámetros espermático fueron evaluados por test Student's y el grupo de ovocitos fueron comparados con test Chi cuadrado un valor de $\mathrm{P}<$ 0.05 fue considerado como siignificativo.

\section{Resultados}

\section{Efecto de la pentoxifilina sobre el movimiento espermático}

Las características espermáticas de los dos grupos normo y oligoastenospérmicos son detallados en la tabla 1. Después de selección percoll la media + SD de formas móviles de los grupos $1,2 \mathrm{a}$ y $2 \mathrm{~b}$ fue $(29.80 \pm 18.20 ; 8.02$ \pm 6.11 y $1.91 \pm 2.0$ respectivamente). La diferencia de velocidad curvilínea (VCL) de normospérmicos y oligoastenospérmicos fue cerca a la significancia a TO y $\mathrm{T} 3(\mathrm{P}=0.061$ y $\mathrm{P}=0.068$ respectivamente $)$. La diferencia de velocidad progresiva (VSL) fue significativa a TO y T3 así como también la velocidad del trayecto (VAP) a TO (Figura 1).

La diferencia de VSL a T3 fue también con tendencia a la significancia $(P=0.054)$. Los valores de los otros parámetros no fueron diferentes.

Estos parámetros de movimiento no se modificaron cuando los espermatozoides de pacientes normospérmicos y severamente oligoastenospérmicos fueron incubados por 40 minutos con $3.6 \mathrm{mM}$ de pentoxifilina. Unicamente se encontró un incremento significativo del desplazamiento lateral de la cabeza (ALH) de pacientes moderadamente oligoastenospérmicos (grupo 2a) a T3 comparado con el control. $(3.31 \pm 0.81 \mu \mathrm{m}$ vs. $4.07 \pm 1.06 \mu \mathrm{m}, \mathrm{P}<$ 0.05 ).

Como se observa en la figura 2, el incremento de ALH por la pentoxifilina fue mayor a $0.5 \mu \mathrm{m}$ en 2 casos de los 14 pacientes.

Tabla 1

CARACTERISTICAS ESPERMATICAS DE PACIENTES INCLUIDOS EN EL ESTUDIO

\begin{tabular}{|c|c|c|c|c|c|c|}
\hline Pacientes & $\begin{array}{c}\text { Volumen de } \\
\text { eyaculado } \\
(\mathrm{ml})\end{array}$ & $\begin{array}{l}\text { Concentración } \\
\times 18^{4} \mathrm{ml}^{-1}\end{array}$ & $\begin{array}{c}\text { Motilidad } \\
\text { normal } \\
(\%)\end{array}$ & $\begin{array}{c}\text { Morfología } \\
(\%)\end{array}$ & $\begin{array}{c}\text { Vitalidad } \\
(\%)\end{array}$ & $\begin{array}{c}\text { Número de } \\
\text { espermatozoides } \\
\text { móviles } \\
\text { x18 }^{6} \mathrm{ml}^{-1}\end{array}$ \\
\hline $\begin{array}{l}\text { grupo } 1 \\
(n=10)\end{array}$ & $3.2 \pm 8.8$ & $63.7 \pm 23.2$ & $56.5 \pm 18.8$ & $56.5 \pm 8.8$ & $83.1 \pm 5.6$ & $36.4 \pm 16.6$ \\
\hline $\begin{array}{l}\text { grupo } 2 a \\
(n=14)\end{array}$ & $3.8 \pm 1.5$ & $26.8 \pm 18.4$ & $32.9 \pm 13.1$ & $35.1 \pm 15.1$ & $74.8 \pm 4.6$ & $7.8 \pm 1.6$ \\
\hline $\begin{array}{l}\text { grupo } 2 b \\
(n=22)\end{array}$ & $4.2 \pm 1.5$ & $7.2 \pm 8.5$ & $30.5 \pm 12.6$ & $21.7 \pm 11.6$ & $61.5 \pm 27.9$ & $1.6 \pm 1.1$ \\
\hline
\end{tabular}

Esta tabla reporta la media $+/-\mathrm{SD}$ de las características espermáticas de los tres grupos del estudio:

Grupo 1: (>10.10 espermatozoides móviles $/ \mathrm{ml})$;

Grupo 2a: (>5 a $£ 10.10^{6}$ espermatozoides móviles $/ \mathrm{ml}$ );

Grupo 2b: ( $£ 5.10^{6}$ espermatozoides móviles $/ \mathrm{ml}$ ). 
Figura 1

PARAMETROS DE MOVIMIENTO DESPUES DE SELECCION EN GRADIENTE DE PERCOLL

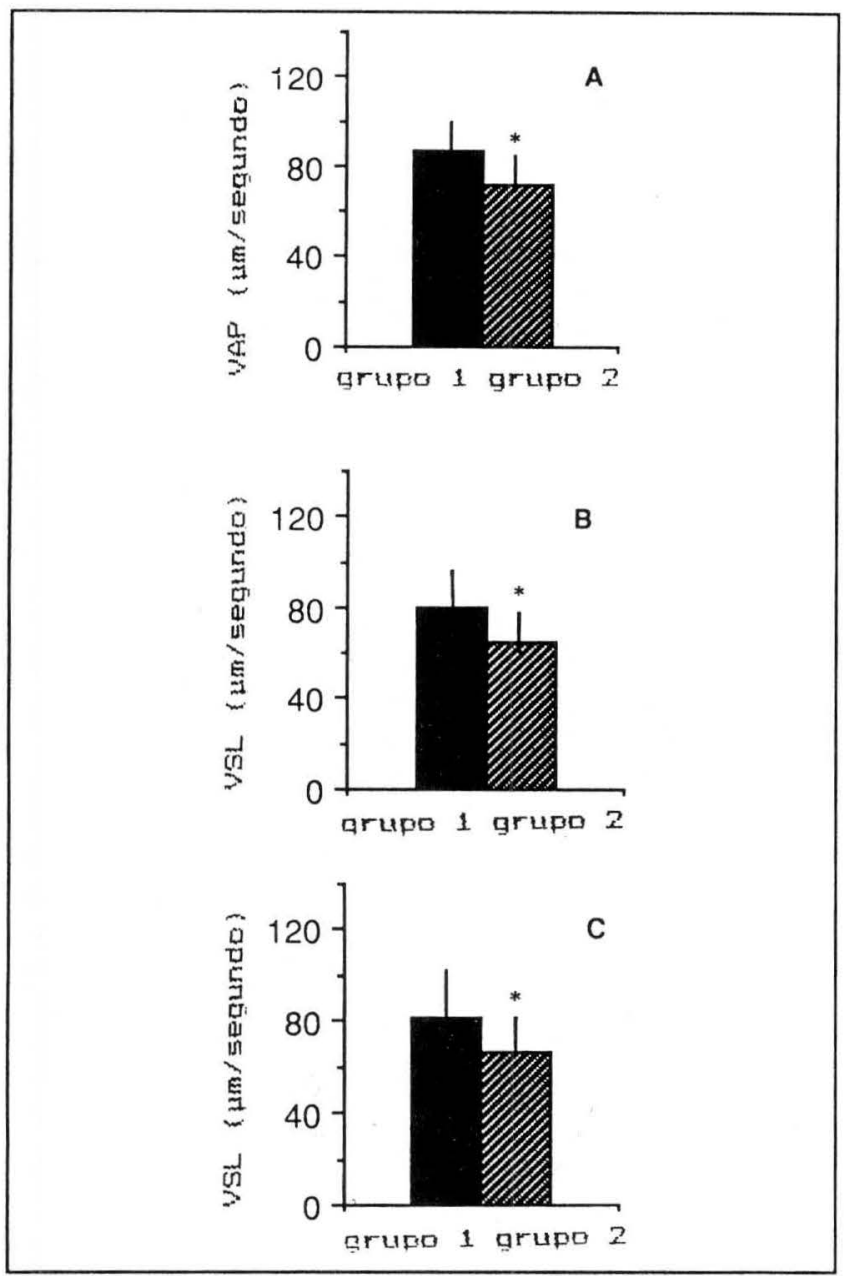

$=$ Pacientes normospérmicos

$=$ Pacientes oligoastenospérmicos

(A) Velocidad de trayecto (VAP) a TO * p $<0.05$

(B) Rectitud (VSL) a TO * p $<0.01$ y

(C) VSL a T3* p $<0.05$

\section{Efecto de la pentofixilina sobre la capacidad fecundante}

Los resultados de fertilización in vitro y de SUZI son reportados en la tabla 2. La taza de fertilización después de usar semen tratado con petoxifilina en FIV fue similar a los resultados cuando se utilizó semen no tratado con pentoxifilina ( $47.1 \%$ vs. $49.3 \%$ respectivamente). Similares resultados se observaron después de SUZI $(16.6 \%$ vs. $5.3 \%$ ).

Un total de 70 embriones fueron obtenidos (35 después de selección percoll y 35 con pentoxifilina), de los cuales 31 embriones fueron transferidos y 33 congelados.

Cuatro embarazos se obtuvieron (dos embarazos después de únicamente selección percoll y dos después de pentoxifilina), el aumento de ALH $(>0.4 \mu \mathrm{m})$ después de utilizar pentoxifilina a TO y T3 no modificó la taza de fertilización.
Figura 2

EFECTO DE LA INCUBACION CON

PENTOXIFILINA SOBRE ALH DE PACIENTES

OLIGOASTENOSPERMICOS DEL GRUPO 2A

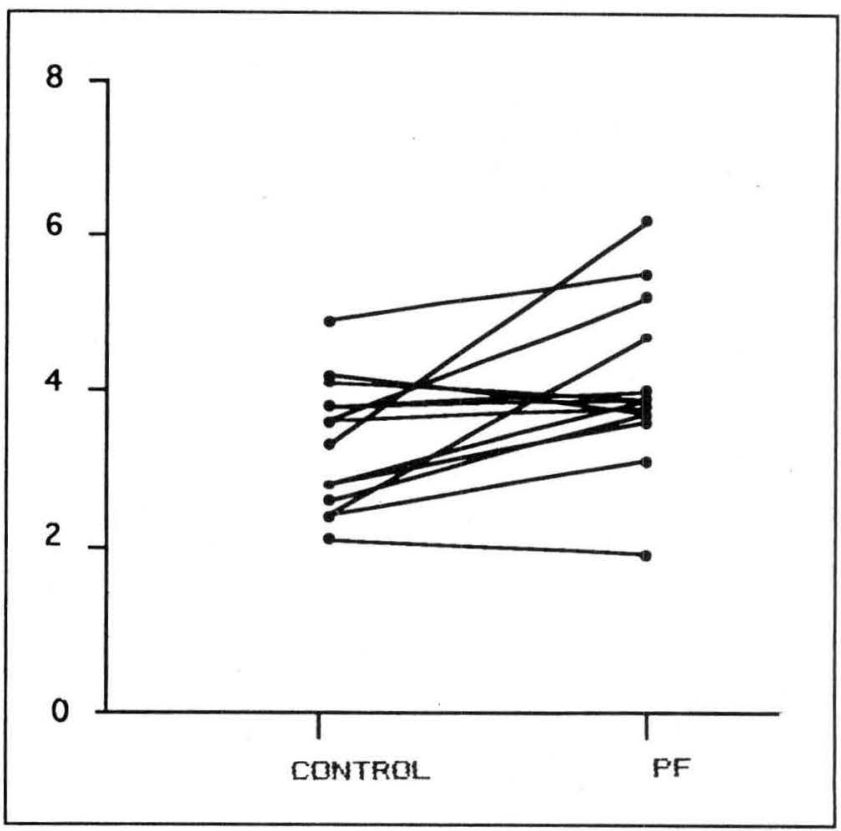

Modificación de la medida de ALH de pacientes moderadamente oligoastenospérmi$\cos 3$ horas después de removida la pentoxifilina.

\section{Discusión}

La pentoxifilina produjo un aumento significativo del desplazamiento lateral de la cabeza (ALH) únicamente en el grupo de pacientes moderadamente oligoastenospérmicos. Como reportado previamente, este efecto fue aún encontrado tres horas después de ser removida la pentoxifilina (3-5). Lewis et al. (4) quien estudió los efectos de la pentoxifilina sobre la motilidad espermática en pacientes normospérmicos reporta que el efecto más importante fue el aumento de ALH sin ningún cambio en el número de espermatozoides móviles progresivos, pero además el semen había sido preparado por swim-up. Rees et al. (15) reportó únicamente un leve aumento de ALH como efecto de la pentoxifilina sobre espermatozoides lavados de donantes normospérmicos.

En nuestro estudio, los pacientes incluidos en el programa de FIV y SUZI presentaban severa oligoastenospermia con una media de $<5 \times 10^{6}$ espermatozoides móviles/ml y la coincubación con pentoxifilina no mejoró los resultados a los obtenidos con percoll.

Nosotros no obtuvimos los resultados reportados por Yovich et al. (8) quien demostró un moderado incremento de la taza de fertilización después de la incubación con pentoxifilina. Esto puede ser explicado con el uso del gradiente de percoll para seleccionar mejor los espermatozoides que por el método de swim-up y la inseminación en microgotas de $30 \mu \mathrm{l}$. En efecto con este tratamiento, la taza de fertilización que nosotros obtuvimos después de inseminación sin pentoxifilina es comparable a la reportada por Yovich con pentoxifilina $(2,8)$. 
Tabla 2

TAZA DE FERTILIZACION DESPUES DE FIV CON INSEMINACION STANDARD E INSEMINACION SUBZONAL (SUZI) SIN O CON PENTOXIFILINA*

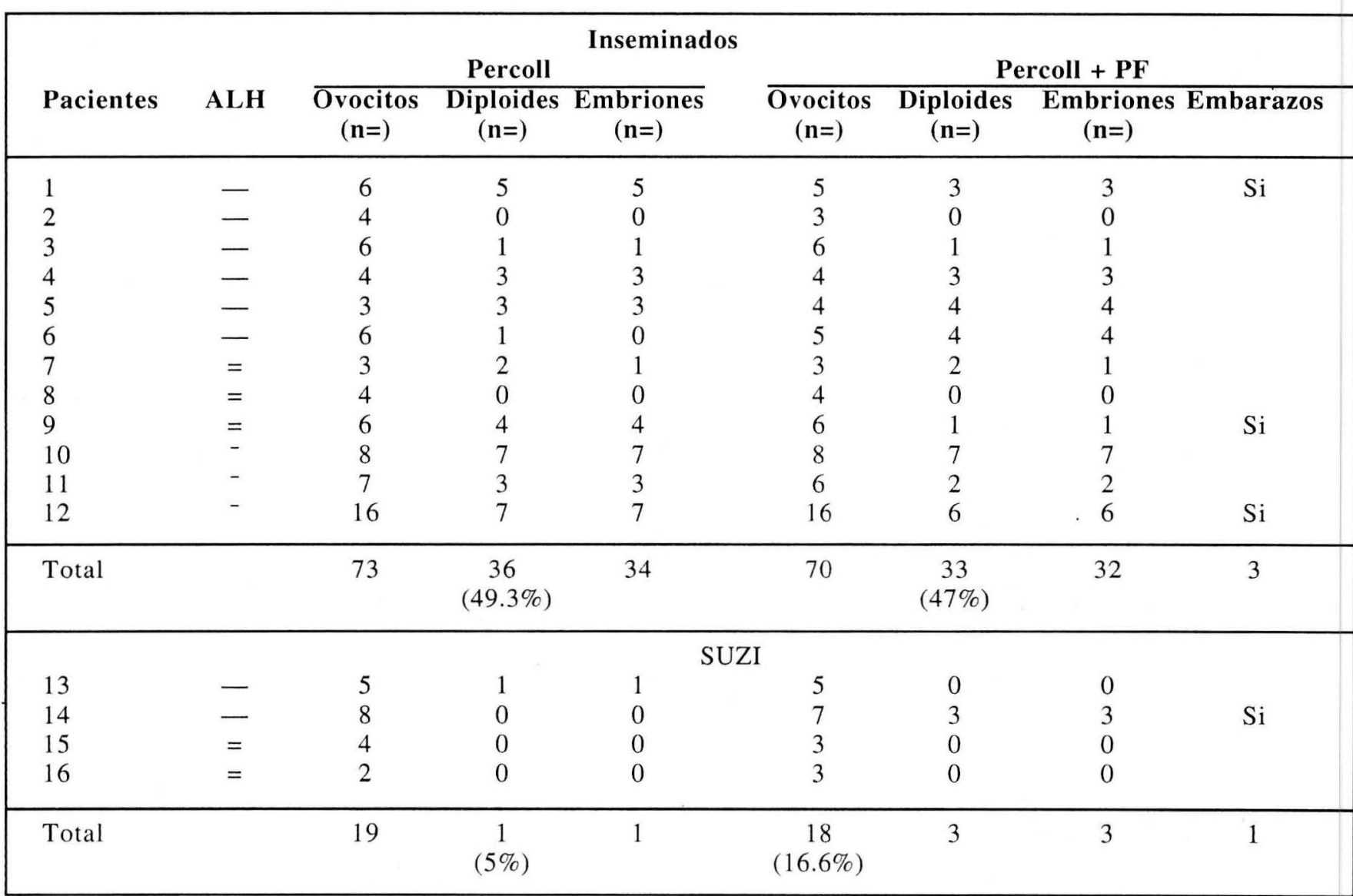

* Valores en paréntesis son porcentajes

$¥$ Diferencias no significativas

- Aumento de ALH ( ${ }^{3} 0.4$ um)

= no cambio de ALH

- Disminución de ALH

PF Pentoxifilina

Sin embargo, Tournaye et al. (9) quien también utilizó migración swim-up después de semen incubado con pentoxifilina y realizó la inseminación en microgotas tuvo una taza de fertilización comparable a la nuestra sin encontrar ningún aumento significativo de la taza de fertilización después de incubación con pentoxifilina.

Las discrepancias de resultados en la literatura puede ser explicada por los diferentes procedimientos de preparación espermática y los protocolos de incubación con la pentoxifilina que han sido utilizados. Algunos estudios han sido hechos con semen lavado, otros con espermatozoides seleccionados por swim-up.

Nosotros analizamos por primera vez los parámetros de movimiento espermático después de selección de espermatozoides por gradiente de percoll y coincubación con pentoxifilina y correlacionamos los hallazgos con los resultados de FIV. Comprobamos nuevamente que la técnica con gradiente de percoll selecciona los mejores espermatozoides (17-18). La inseminación con técnica de microgota puede ser lo ideal para mejorar los resultados de FIV por oligoastenospermia. Tournaye y nosotros, al utilizar esta técnica obtenemos una taza de fertilización relativamente alta la cual no fue modificada con el uso de la pentoxifilina. Fuscaldo et al. reportó el uso de la pentoxifilina y 2 deosyadenosina durante la preparación de espermatozoides para SUZI (16). Ellos utilizando swim-up encontraron un aumento significante de la taza de fertilización a $22.1 \%$. Sin embargo, la taza de fertilización después de SUZI con pentoxifilina es comparable con nuestros resultados de SUZI sin pentoxifilina $(14,19)$.

Todos estos datos permiten sugerir que la selección de espermatozoides con gradiente de percoll permite seleccionar los espermatozoides con mejores parámetros de movimiento y motilidad. La adición de pentoxifilina a esta fracción de espermatozoides seleccionados no presenta ningún efecto. La subsecuente FIV y SUZI no encuentra una mejoría de la taza de fertilización después del uso de la pentoxifilina. Por lo contrario, parece ser más interesante el uso de la pentoxifilina en espermatozoides seleccionados por swim-up. Esta hipótesis la sustentamos con un estudio preliminar de compa- 
rar en tres especímenes el efecto de la pentoxifilina después de swim-up y después de selección por percoll.

En resumen, la pentoxifilina parece tener poco efecto en los parámetros de movimiento sobre espermatozoides seleccionados por percoll. Su uso no modifica el porcentaje de motilidad ni mejora los parámetros de movimiento de pacientes normospérmicos. Sin embargo aumenta el
$\mathrm{ALH}$ en algunos pacientes oligoestenospérmicos. $\mathrm{Pa}-$ cientes a quienes el ALH fue aumentado, no se observó una diferencia significativa en taza de fertilización usando pentoxifilina en comparación a los resultados con percoll. La utilidad de la pentoxifilina en fecundación in vitro por causa masculina deberá ser evaluada en un grupo más amplio.

\section{BIBLIOGRAFIA}

1. Ward A., Clissold SP. Pentoxifylline: a review of its pharmacodynamic and pharmacokinetic properties, and its therapeutic efficiency. Drugs 1987; 34: 50-60.

2. Yovich JM., Edirisinghe WR., Cummins JM., Yovich JL. Preliminary results using pentoxifylline in a pronuclear stage tubal transfer (PROST) program for severe male factor infertility. Fertil Steril 1988; 50: $179-181$.

3. Tesarik J. Thébault A., Testart J. Effect of pentoxifylline on sperm movement characteristics in normazoospermic and asthenozoospermic specimens. Hum Reprod 1992; 7: 1257-1263.

4. Lewis SE., Moohan JM., Thompson W. Effects of pentoxifylline on sperm motility in normospermic individuals using computer-assisted analysis. Fertil Steril 1993; 59: 418-423.

5. Pang SC., Williams DB., Huanq T., Wang C. Effects of pentoxifylline on spermy motility and hyperactivated motility in vitro: a preliminar report. Fertil Steril 1993; 59: 465-467.

6. Barlow P., Delvigne A., Van Dromme J., Van Hoeck J., Vandesboch K., Leroy F. Predictive value of classical and automated sperm analysis for in vitro fertilization. Hum Reprod 1191; 6: 1119-1124.

7. Check JH., Bollendorf A., Lee MA., Nazari A., Nowroozi K. Correlatiion of computarized semen analysis with successful fertilization of oocytes in an in vitro.

8. Yovich JM., Edirishinge WR., Cummings JM., Yovich JL. Influence of pentoxifylline in severe male factor infertility. Fertil Steril 1990; 53: 715-722

9. Tournaye H., Janssens R., Camus M., Staessen C., Devroey P., Van Steirteghem A. Pentoxifylline is not useful in enhancing sperm function in cases with previous in vitro fertilization failure. Fertil Steril 1993; 59: 210-215

10. World Health Organization. Who Laboratory Manual for the Examination of Human Semen and Semen-Cervical Mucus Interaction. 2nd edition. Cambridge, The Press Syndicate of the University of Cambridge, 1987; 28.

11. Jager S., Kremer J., Van Slochterendraaima T. A simple method of screening for antiesperm antibodies in the human male. Int .J. Fertil 1978; 23: 12 .

12. De Almeida M., Soumah A., Jouannet P. Incidence of sperm associated immunoglobulins in infertile men suspected of antisperm autoimmunity. Int. J. Androl. 1986; 9: 321-330.

13. Frydman R., Forman RG., Belaisch-Allart J., Hazout A., Rainhorn JD., Fries N., Testart J. Improvements in ovarian stimulation for in vitro fertilization. Ann NY Acad. Sci. 1988; 541: 30-36.

14. Wolf JPh., Ducot B., Kunstmann JM., Frydman R., Jouannet P. Influence of sperm parameters on outcome of subzonal insemination in the case of previos IVF failure. Hum. Reprod. 1992; 7: 1407-1413.

15. Rees JM., Ford WCL., Hull MGR. Effect of caffeine and of pentoxifylline on the motility and metabolism of human spermatozoa. J. Reprod. Fert. 1990; 90: 147-156.

16. Fuscaldo G. Sobieszczuk D., Trounson AD. Improved fertilization rates following microinjection of human spermatozoa, pretreated with 2 deoxyadenosine and pentoxifylline. 7 th World Congress on In Vitro Fertilization and Assited Procreations, Paris Jun Abstract 1991; 145-146.

17. McClure RD., Nunes L., Tom Ray. Semen manipulation: improved sperm recovery and function with a two-layer Percoll gradient. Fertil Steril 1989; 51: 874-877.

18. Barthelemy C., Fricot G., Hammamah, Lebols C., Lansac J., Tharanne MJ. Ultrastructural comparison of human spermatozoa along a peercoll density gradient. Int. J. Fertil 1992; 37: 362-367.

19. Wolf JPh., Feneux D., Escalier D., Rodrigues D., Frydman R., Jouannet P. Pregnancy after subzonal insemination with spermatozoa lacking outer dynein arms. J. Reprod. Fertil 1993; 97: 487-792. 